\title{
Podocalyxin-like 1 is associated with tumor aggressiveness and metastatic gene expression in human oral squamous cell carcinoma
}

\author{
CHENG-WEI LIN ${ }^{1,2}$, MIN-SIOU SUN ${ }^{2}$ and HAN-CHUNG WU ${ }^{2,3}$ \\ ${ }^{1}$ Department of Biochemistry, School of Medicine, Taipei Medical University; ${ }^{2}$ Institute of Cellular and \\ Organismic Biology, Academia Sinica; ${ }^{3}$ Genomics Research Center, Academia Sinica, Taipei 115, Taiwan, R.O.C.
}

Received January 6, 2014; Accepted March 7, 2014

DOI: $10.3892 /$ ijo.2014.2427

\begin{abstract}
Metastasis-mediated death remains a major challenge in cancer treatment due to the lack of identifiable biomarkers for early diagnosis. Identifying tumor-specific biomarkers is critical for the development of diagnostic and therapeutic tools. In the present study, we found that podocalyxin-like 1 (PODXL), a cell surface glycoprotein, was overexpressed in cancer tissues and was upregulated in lymph node metastatic tumor cells. The expression of PODXL was associated with the migratory ability of human oral squamous cell carcinoma (OSCC). Knockdown of PODXL by small hairpin RNA in the SAS OSCC cell line reduced tumor migration and invasion, and inhibited cell proliferation and colony formation. Suppression of PODXL resulted in downregulation of focal adhesion kinase (FAK) and paxillin phosphorylation. PODXL silencing inhibited filopodia formation, and suppressed F-actin and cortactin colocalization. In addition, PODXL expression was associated with the DNA methylation status, and treatment with the DNA methyltransferase inhibitor 5-aza-deoxycytidine increased the PODXL transcriptional level. Moreover, DNA microarray analysis data revealed that suppression of PODXL significantly affected subsets of genes associated with extracellular matrix organization, the epithelial-mesenchymal transition, and the expression of metastasis-related cytokines. Collectively, these data showed that the overexpression of PODXL may be associated with tumor aggressiveness and that PODXL could be a diagnostic biomarker for metastatic OSCC.
\end{abstract}

Correspondence to: Dr Cheng-Wei Lin or Professor Han-Chung Wu, Institute of Cellular and Organismic Biology, Academia Sinica, Taipei 115, Taiwan, R.O.C.

E-mail: cwlin@tmu.edu.tw

E-mail: hcw0928@gate.sinica.edu.tw

Key words: podocalyxin-like 1, human oral squamous cell carcinoma, invasion, metastasis, epigenetic regulation

\section{Introduction}

The incidence of human oral squamous cell carcinoma (OSCC) is increasing rapidly over the years. On average, 5\% of all cancers diagnosed annually in the Western countries are OSCC, whereas in the Asian countries, especially in India, Taiwan, and the Philippines, the incidence rate of OSCC is $\sim 10-50 \%$ due to the popularity of tobacco, betel nut, and alcohol consumption in these regions $(1,2)$. Surgery combined with chemotherapy or radiotherapy has improved the survival rate. However, metastatic OSCC, which accounts for almost $80 \%$ of all OSCC patients, is the major cause of death among cancer patients. Metastatic OSCC, which invades through cervical lymph nodes, presents a difficult challenge for surgical therapy and often results in recurrence and death. Therefore, identifying reliable biomarkers would allow early diagnosis of the disease, and hence improve the prognosis for the patients.

Podocalyxin-like 1 (also known as gp135, PCLP and PODXL) is a cell surface glycoprotein belonging to the CD34 family including CD34, podocalyxin-like 1 (PODXL) and endoglycan (PODXL2). CD34 is predominantly expressed in hematopoietic stem cells and vascular endothelial cells $(3,4)$. Podocalyxin-like 1 (PODXL) was initially identified in kidney glomerular epithelial cells (5), and was also found in hematopoietic progenitor cells and vascular endothelial cells (6). The protein domains of CD34 and PODXL are similar, which include an $O$-glycosylated and sialylated enriched extracellular domain, a transmembrane domain, and a short cytoplasmic domain for docking of proteins with the PDZ domain, are similar in structures (7). Proteins with a PDZ domain, such as Ezrin-binding protein (EBP) and the $\mathrm{Na}^{+} / \mathrm{H}^{+}$exchanger regulatory factor (NHERF), often participate in cytoskeletal rearrangement, implying that PODXL may have a function in cellular morphogenesis. The cellular function of PODXL was reported to support the structure of the podocyte basal surface and the formation of a preapical domain during polarization (8). The binding of PODXL to Ezrin or NHERF induces activation of small GTPase RhoA and Rac1, and facilitates actin reorganization (9-11). Dissociation of PODXL from actin results in loss of glomerular foot formation and podocyte integrity, and the absence of PODXL leads to perinatal lethality (12). In tumor cells, abnormal expression of PODXL was reported to produce 
anti-adhesion and characteristics of aggressiveness in a variety of cancers. Overexpression of PODXL is associated with lymphatic invasion of breast cancer (13) and poor prognosis in colorectal, bladder and brain tumors (14-16). Despite the critical role it plays in cancer metastasis, the exact mechanism of PODXL in OSCC is still unclear.

Epigenetic regulation, including DNA methylation and histone modification, plays important roles in gene modulation. DNA methylation of promoter $\mathrm{CpG}$ islands regulates transcriptional activation and repression. Dysregulation of the DNA methylation status alters the transcription activity of oncogenes and tumor suppressor genes, which results in abnormalities in cellular behavior, and eventually contributes to neoplastic formation (17). Aberrant changes in DNA methylation were reported with malignant progression in OSCC (18). Promoter hypermethylation of ALK in OSCC was correlated with node-negative metastasis (19). A global methylation analysis of OSCC revealed an aberrant methylation status enriched in genes often found in the WNT and mitogenactivated protein kinase (MAPK) signaling pathway (20), yet the DNA methylation of PODXL and its expression in association with tumor aggressiveness in OSCC are still unclear. In the present study, we investigated the role of PODXL in contributing to tumor metastasis in human OSCC. We found that PODXL expression was associated with tumor aggressiveness and invasiveness. PODXL regulates the phosphorylation of focal adhesion kinase (FAK) and paxillin, and the formation of filopodia and invadopodia. PODXL expression was associated with the DNA methylation status. Modulation of the extracellular matrix (ECM) and pro-metastatic gene expression levels by PODXL contribute to tumor metastasis.

\section{Materials and methods}

Cell culture. Human OSCC lines (FaDu and SAS) were purchased from American Type Culture Collection (ATCC; Manassas, VA, USA). SAS cells were grown in Dulbecco's modified Eagle's medium (DMEM), and FaDu cells were grown in RPMI-1640. All culture media were supplemented with 5\% fetal bovine serum (FBS, Gibco, Grand Island, NY, USA) and penicillin/streptomycin (Gibco), and were grown at $37^{\circ} \mathrm{C}$ in a $5 \% \mathrm{CO}_{2}$ atmosphere.

Lentivirus production. Small hairpin RNA vectors for PODXL silencing (5'-GTCGTCAAAGAAATCACTATT-3') were obtained from the National RNAi Core Facility (Academia Sinica, Taiwan). To generate stable PODXL-knockdown cell lines, HEK293T packaging cells were co-transfected with a packaging plasmid (pCMV- $\triangle \mathrm{R} 8.91$ ), and envelope (pMDG) and hairpin pLKO-RNAi vectors using a PolyJET Transfection kit (SignaGen Laboratories, Ijamsville, MD, USA). At 48-h posttransfection, virus-containing supernatants were collected, mixed with fresh media containing polybrene $(8 \mu \mathrm{g} / \mathrm{ml})$, and incubated with target cells for another $48 \mathrm{~h}$. Transduced cells were selected with puromycin $(2 \mu \mathrm{g} / \mathrm{ml})$ for 7 days.

Transwell migration and invasion assays. Cells $\left(10^{5}\right)$ were seeded in a Transwell insert (8- $\mu \mathrm{m}$ filters, Corning, New York, NY, USA) coated with or without Matrigel (BD Biosciences, La Jolla, CA, USA) for 8 (for the migration assay) and $24 \mathrm{~h}$ (for the invasion assay). After incubation, cells were fixed with $4 \%$ paraformaldehyde for $10 \mathrm{~min}$. Cells that had not invaded were removed with a cotton swab; invaded cells were stained with 4',6-diamino-2-phenylindole (DAPI), imaged under an inverted fluorescent microscope (Zeiss), and quantified using ImageJ software.

Time-lapse migration assay. Cells $\left(2 \times 10^{4}\right)$ were seeded in a chamber with complete growth media, and monitored under an inverted light microscope (Zeiss HAL100 reflected-light microscope) with a temperature and $\mathrm{CO}_{2}$ control system. Images were captured every $10 \mathrm{~min}$ for $6 \mathrm{~h}$. The migration distance was defined as the movement of the cell center per unit time, as measured by MetaMorph software.

Cell proliferation assay. Differences in the proliferation of SAS/LKO and SAS/shPODXL cells were evaluated by a 3-(4,5-dimethylthiazol-2-yl)-2,5-diphenyl tetrazolium bromide (MTT) assay. Briefly, 2,000 cells were seeded in a 96-well plate with complete media for 1-5 days, and cells were incubated in $50 \mu \mathrm{l}$ of $0.5 \mathrm{mg} / \mathrm{ml} \mathrm{MTT}$ for $3 \mathrm{~h}$. DMSO was added to dissolve the crystals, which were measured on a microplate reader at 540-nm absorbance. Data are presented as the percentage of growth on the day after seeding. To test the effect of PODXL on colony formation, 2000 SAS/LKO and SAS/shPODXL cells were seeded in 6-well plates and incubated for 7 days. Surviving colonies were stained with crystal violet after methanol fixation. Visible colonies $(\geq 50$ cells) were counted.

Western blotting. Cells were lysed in RIPA buffer $(150 \mathrm{mM}$ $\mathrm{NaCl}, 1 \%$ Nonidet P-40, $0.5 \%$ sodium deoxycholate, $0.1 \%$ sodium dodecylsulfate (SDS), and $50 \mathrm{mM}$ Tris- $\mathrm{HCl}$ at $\mathrm{pH} 7.4$ ) containing a protease and phosphatase inhibitor cocktail (Roche, Basel, Switzerland). For immunoprecipitation, cell lysates were precleared with agarose-protein $\mathrm{G}$ for $1 \mathrm{~h}$ and incubated overnight at $4^{\circ} \mathrm{C}$ with the appropriate protein $\mathrm{G}$-conjugated primary antibodies. Beads were washed three times with RIPA buffer and boiled in sample buffer $(50 \mathrm{mM}$ Tris- $\mathrm{HCl}$ at $\mathrm{pH} 6.8,2 \% \mathrm{SDS}, 0.1 \%$ bromophenol blue, and $10 \%$ glycerol). Equal amounts of proteins were separated on SDS-polyacrylamide gel electrophoresis (PAGE) and then transferred to polyvinylidene difluoride membranes (Millipore, Bedford, MA, USA). Membranes were blocked with $1 \%$ bovine serum albumin (BSA)/TBST and incubated overnight with specific primary antibodies against PODXL (1:500, Santa Cruz Biotechnology, Santa Cruz, CA, USA), FAK (1:2000, Millipore), phospho-FAK (1:2000, Millipore), paxillin (1:2000, Millipore), phospho-paxillin (1:500, Millipore), or $\alpha$-tubulin (1:5000, Sigma, St. Louis, MO, USA). Membranes were then incubated with the appropriate horseradish peroxidase (HRP)-conjugated secondary antibodies (1:10000, Jackson Immunoresearch) for $1 \mathrm{~h}$ at room temperature, and proteins were detected using an ECL kit (Millipore, Temecula, CA, USA).

Immunofluorescence. Cells were grown onto cover slide and fixed with $4 \%$ paraformaldehyde for $10 \mathrm{~min}$, followed by blocking with $3 \%$ bovine serum albumin (BSA) for $1 \mathrm{~h}$. The cover slide was incubated with FITC-conjugated cortactin 
Table I. List of oligonucleotides for real-time PCR assay.

\begin{tabular}{|c|c|c|}
\hline $\begin{array}{l}\text { Gene } \\
\text { symbol }\end{array}$ & Name & Sequence $\left(5^{\prime} \rightarrow 3^{\prime}\right)$ \\
\hline PODXL & Podocalyxin-like 1 & $\begin{array}{l}\text { F: aaggccaggggttcacat } \\
\text { R: agcctcgcatccetctaact }\end{array}$ \\
\hline$L O X$ & Lysyl oxidase & $\begin{array}{l}\text { F: tgggaatggcacagttgtc } \\
\text { R: aaacttgctttgtggcettc }\end{array}$ \\
\hline LOXL4 & Lysyl oxidase-like 4 & $\begin{array}{l}\text { F: ccagcttctgtctggaggac } \\
\text { R: aagttggcacatgcgtagc }\end{array}$ \\
\hline COL17A1 & Collagen type XVII, $\alpha 1$ & $\begin{array}{l}\text { F: gctggagatctggattacaatga } \\
\text { R: ccttgcagtaggecctga }\end{array}$ \\
\hline COL13A1 & Collagen type XIII, $\alpha 1$ & $\begin{array}{l}\text { F: taagcagcatgccagcag } \\
\text { R: cagtcgcactgaattgagga }\end{array}$ \\
\hline ITGB3 & Integrin $\beta 3$ & $\begin{array}{l}\text { F: cgctaaatttgaggaagaacg } \\
\text { R: gaaggtagacgtggcctcttt }\end{array}$ \\
\hline $\mathrm{CDHI}$ & E-cadherin & $\begin{array}{l}\text { F: ggaactatgaaaagtgggcttg } \\
\text { R: aaattgccaggctcaatgac }\end{array}$ \\
\hline $\mathrm{CDH} 3$ & P-cadherin & $\begin{array}{l}\text { F: caccacccaccctgagag } \\
\text { R: tttggcetcaaaatccaaac }\end{array}$ \\
\hline CDH11 & OB-cadherin & $\begin{array}{l}\text { F: aaacagcctggctcaacatc } \\
\text { R: cttcctgatgccgattgtg }\end{array}$ \\
\hline CREM & $\begin{array}{l}\text { cAMP responsive } \\
\text { element modulator }\end{array}$ & $\begin{array}{l}\text { F: tgatggcacacagcagttct } \\
\text { R: atgtcaccagtggcagctt }\end{array}$ \\
\hline$M M P 1$ & $\begin{array}{l}\text { Matrix } \\
\text { metalloproteinase } 1\end{array}$ & $\begin{array}{l}\text { F: gctaacctttgatgctataactacga } \\
\text { R: tttgtgcgcatgtagaatctg }\end{array}$ \\
\hline$I L 1 B$ & Interleukin $1 \beta$ & $\begin{array}{l}\text { F: tacctgtcctgcgtgttgaa } \\
\text { R: tctttgggtaatttttgggatct }\end{array}$ \\
\hline ILIA & Interleukin $1 \alpha$ & $\begin{array}{l}\text { F: acaaaaggcgaagaagactga } \\
\text { R: ggaactttggccatcttgac }\end{array}$ \\
\hline IL8 & Interleukin 8 & $\begin{array}{l}\text { F: agacagcagagcacacaagc } \\
\text { R: atggttccttccggtggt }\end{array}$ \\
\hline IL24 & Interleukin 24 & $\begin{array}{l}\text { F: gaagaattgaggctgcttgg } \\
\text { R: gagggcagaagggtctgg }\end{array}$ \\
\hline TIMP1 & $\begin{array}{l}\text { Tissue inhibitor of } \\
\text { metalloproteinase } 1\end{array}$ & $\begin{array}{l}\text { F: gggcttcaccaagacctaca } \\
\text { R: tgcaggggatggataaaca }\end{array}$ \\
\hline CCNA2 & Cyclin A2 & $\begin{array}{l}\text { F: ggtactgaagtccgggaacc } \\
\text { R: gaagatccttaaggggtgcaa }\end{array}$ \\
\hline$O D C 1$ & $\begin{array}{l}\text { Ornithine } \\
\text { decarboxylase } 1\end{array}$ & $\begin{array}{l}\text { F: aaaacatgggcgcttacact } \\
\text { R: tggaattgctgcatgagttg }\end{array}$ \\
\hline CCNE2 & Cyclin E2 & $\begin{array}{l}\text { F: gccattgattcattagagttcca } \\
\text { R: ctgtcccactccaaacctg }\end{array}$ \\
\hline$G A P D H$ & $\begin{array}{l}\text { Glyceraldehyde-3- } \\
\text { phosphate dehydrogenase }\end{array}$ & $\begin{array}{l}\text { F: cttcaccaccatggaggaggc } \\
\text { R: ggcatggactgtggtcatgag }\end{array}$ \\
\hline $18 s r R N A$ & & $\begin{array}{l}\text { F: gcaattattccccatgaacg } \\
\text { R: gggacttaatcaacgcaagc }\end{array}$ \\
\hline
\end{tabular}

(Merck Millipore) and rhodamine-conjugated phalloidin (Invitrogen) for $1 \mathrm{~h}$ at room temperature. The fluorescent images were observed under a confocal microscope (TCS-SP5, Leica).
Immunohistochemical (IHC) analysis of PODXL protein. A paraffin-embedded human colon cancer tissue microarray (US Biomax Inc.) was immersed in xylene for $30 \mathrm{~min}$ and then rehydrated in graded ethanol. The slide was immersed with $0.01 \mathrm{M}$ sodium citric buffer ( $\mathrm{pH}$ 6.0) for antigen retrieval, followed by soaking with $3 \%$ hydrogen peroxide and blocking with normal horse serum (ABC kit, Vector). The slide was incubated with anti-PODXL antibody (10 $\mu \mathrm{g} / \mathrm{ml}$, Sigma) for $1 \mathrm{~h}$ at room temperature. After washing three times with PBST, the Super Enhancer reagent (Super Sensitive Polymer HRP detection system, BioGenex) was added and incubated for $20 \mathrm{~min}$. DAB Chromogen was then added for $3 \mathrm{~min}$, and the reaction was stopped by PBS washing. The slide was stained by hematoxylin for $3 \mathrm{~min}$ and mounted with a xylene-based mounting solution.

Detection of PODXL methylation. The methylation status in PODXL was analyzed by a methylation-specific polymerase chain reaction (MSP) assay using an EZ DNA MethylationDirect assay kit (Zymo Research). Briefly, the genomic DNA of SAS and FaDu cells was extracted and modified by sodium bisulfate treatment, which converted all of the unmethylated cytosines to uracils while leaving methylated cytosines unchanged. Bisulfate-converted DNA was subjected to subsequent PCR amplification. The specific MSP primers for PODXL (methylated, PODXL-M: 5'-TCGTCGGGTTTAT TTAGAAGTATTC-3' and 5'-TATATATACGCGAAAA CCAAAACG-3') and (unmethylated, PODXL-U: 5'-TGT TGGGTTTATTTAGAAGTATTTGG-3' and 5'-TATATATA CACAAAAACCAAAACAA-3') were designed from the MethPrimer database. Methylated and unmethylated genomic DNAs (Zymo Research) were used as an experimental control. To analyze the methylated $\mathrm{CpG}$ site in the PODXL promoter region, specific PCR primers designed from the MethPimer database were used for amplification, and PCR products were cloned into the pGEM-T vector for further sequencing analysis. Four clones were sequenced to assess the level of methylation of each $\mathrm{CpG}$ site.

Real-time PCR. Total RNA was extracted using an RNeasy Plus Mini kit (Qiagen) and reverse-transcribed using SuperScrip III reverse transcriptase (Invitrogen). A quantitative PCR was performed using the resulting cDNA, LightCycler 480 SYBR Green I Master Mix (Roche) and a LightCycler 480 System (Roche). Results are expressed as multiples of change relative to the control sample, using the method of $\triangle \triangle \mathrm{C}_{\mathrm{T}}$. GAPDH or $18 S r R N A$ was used as internal control for normalization. Primer sequences are listed in Table I.

cDNA microarray and bioinformatic analysis. Total RNA was extracted using an RNeasy Plus Mini kit (Qiagen), and RNA quality was analyzed on an Agilent 2100 Bioanalyzer (RNA6000 nanochip). A human cDNA microarray was analyzed according to the protocol of the Agilent 2 color system (Agilent G4845A, 4x44K). Experiments were performed and analyzed by the Microarray Core Facility of the Institute of Molecular Biology, and the Core Facility of the Institute of Cellular Organismic and Biology, Academia Sinica. Genetic networks were analyzed using Pathway Studio 7 and Sub-network Enrichment Analysis (SNEA) software (Ariadne 
Colon: cancer vs. normal

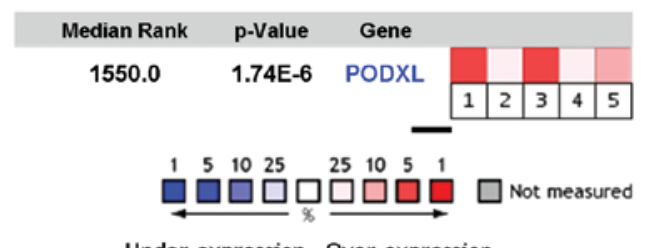

Under-expression Over-expression
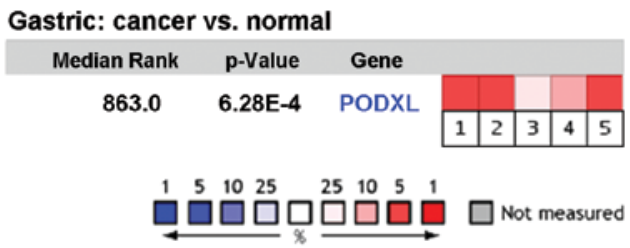

Under-expression Over-expression

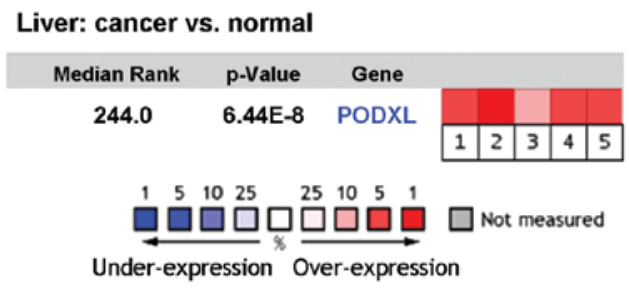

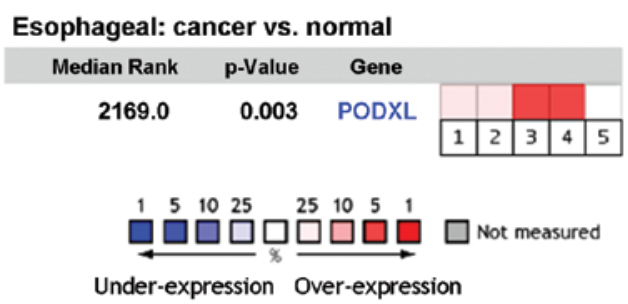

Head and Neck: cancer vs. normal

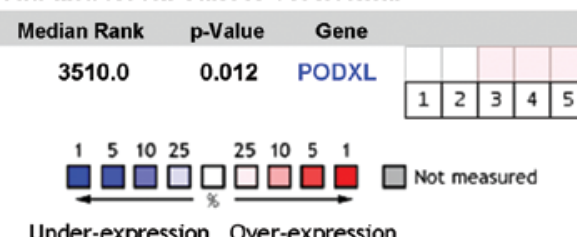

Under-expression Over-expression

Figure 1. Comparison of PODXL expression in cancerous vs. normal tissues of different cancer types. The Oncomine Cancer Profiling Database was used to assess the expression level of PODXL in cancer vs. normal tissues. Each box represents a dataset, which includes colon [1, Hong (21); 2, Kaiser (22); 3, Ki et al (23); 4, Sabates-Bellver (24); 5, Skrzypczak (25)], gastric [1, Cho (26); 2, D'Errico (27); 3, TCGA_dataset; 4, TCGA_dataset; 5, Wang (28)], liver [1, Chen (29); 2, Mas (30); 3, Roessler (31); 4, Roessler (31); 5, Wurmbach (32)], esophageal [1, Hao (34); 2, Hu (35); 3, Kim (36); 4, Kimchi (37); 5, Su (33)], head and neck [1, Cromer (38); 2, Ginos (39); 3, Schlingemann (40); 4, Sengupta (41); 5, Toruner (42)] (see refs. 21-42). The p-value is given for the medium-rank analysis.

Genomics, Rockville, MD, USA). Individual cancer datasets were downloaded from Oncomine (Compendia Bioscience). p-values are given for the medium-rank analysis.

Statistical analysis. The data were derived from at least three independent experiments. Values are expressed as the mean \pm standard error of the mean (SEM). Significant differences were determined using a two-tailed, unpaired t-test, unless otherwise specified. A p-value of $<0.05$ or $<0.01$ was considered significant.

\section{Results}

PODXL is overexpressed in cancer cells and is associated with tumor aggressiveness in OSCC. In order to investigate the association of PODXL with cancer, we used the Oncomine Cancer Profiling database to analyze expression levels of PODXL in cancerous vs. normal tissues. Five types of cancer were selected, and five array datasets were analyzed in each cancer type including colon (21-25), gastric (26-28), liver (29-32), esophageal (33-37), and head and neck cancers (38-42). Results showed that PODXL was overexpressed in all types of cancerous tissues, compared to normal ones (Fig. 1), suggesting that PODXL expression may be associated with cancer. Assessment of the PODXL messenger RNA level by real-time PCR analyses showed similar results in colon cancer specimens (Fig. 2A). Moreover, results of the immunohistochemical assay revealed that the PODXL protein level was upregulated in lymph node metastatic colon tumor tissues, compared to matched primary tumor sites $(n=33, p=0.022)$ (Fig. 2B).
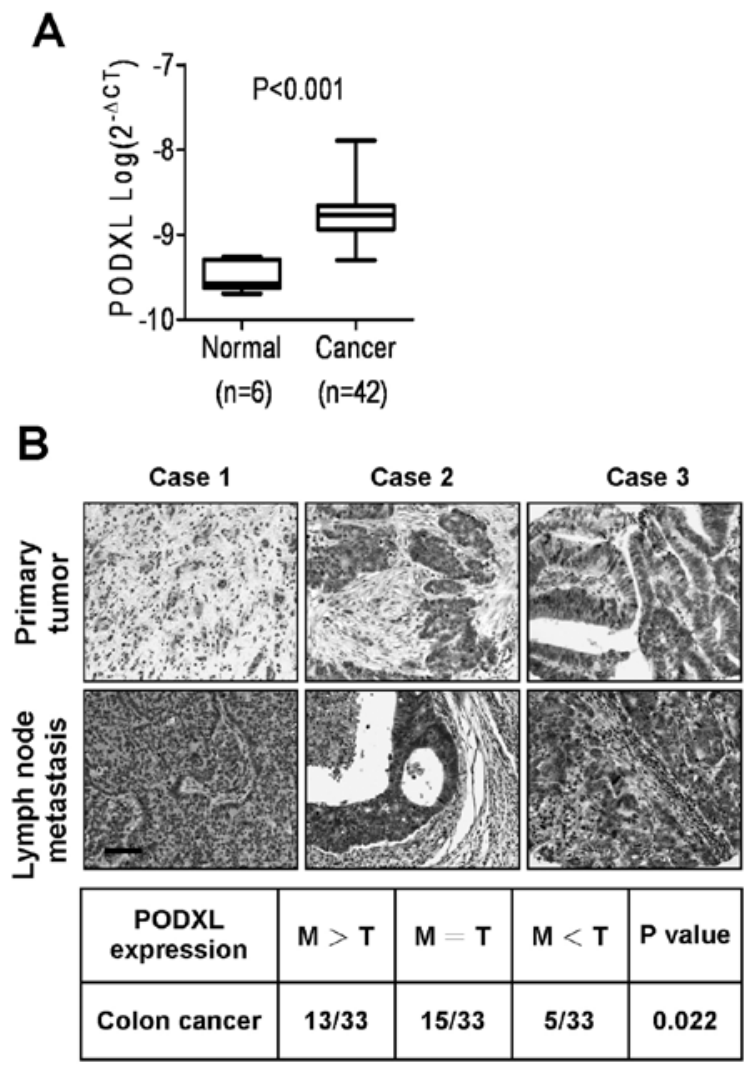

Figure 2. Overexpression of PODXL is associated with cancer malignancy (A) A real-time PCR analysis of PODXL transcription in normal and cancerous colon tissues. Representative data show the $\log \left(2^{-\mathrm{ACT}}\right)$ value. The p-value was analyzed by a Mann-Whitney test. (B) Immunohistochemical analysis of the PODXL protein level in primary and matched metastatic tumor tissues. The p-value was analyzed by a Chi-squared test. 

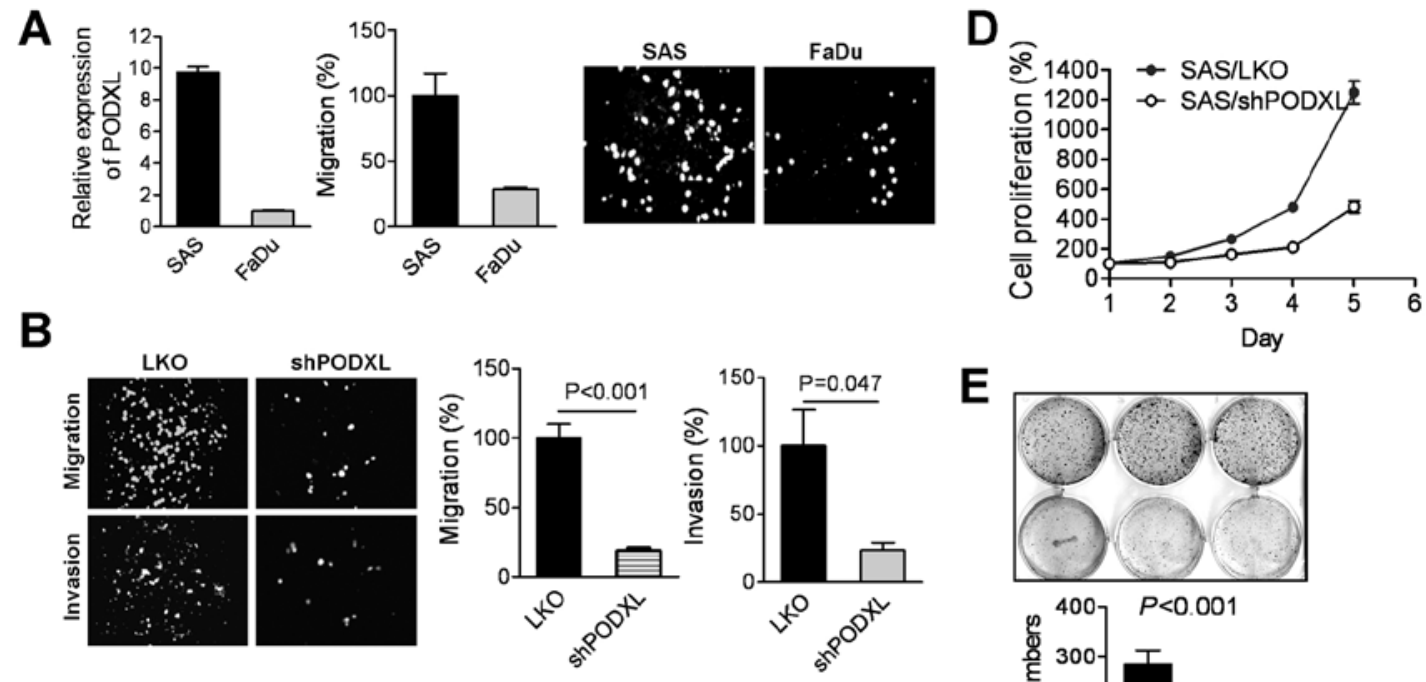

E
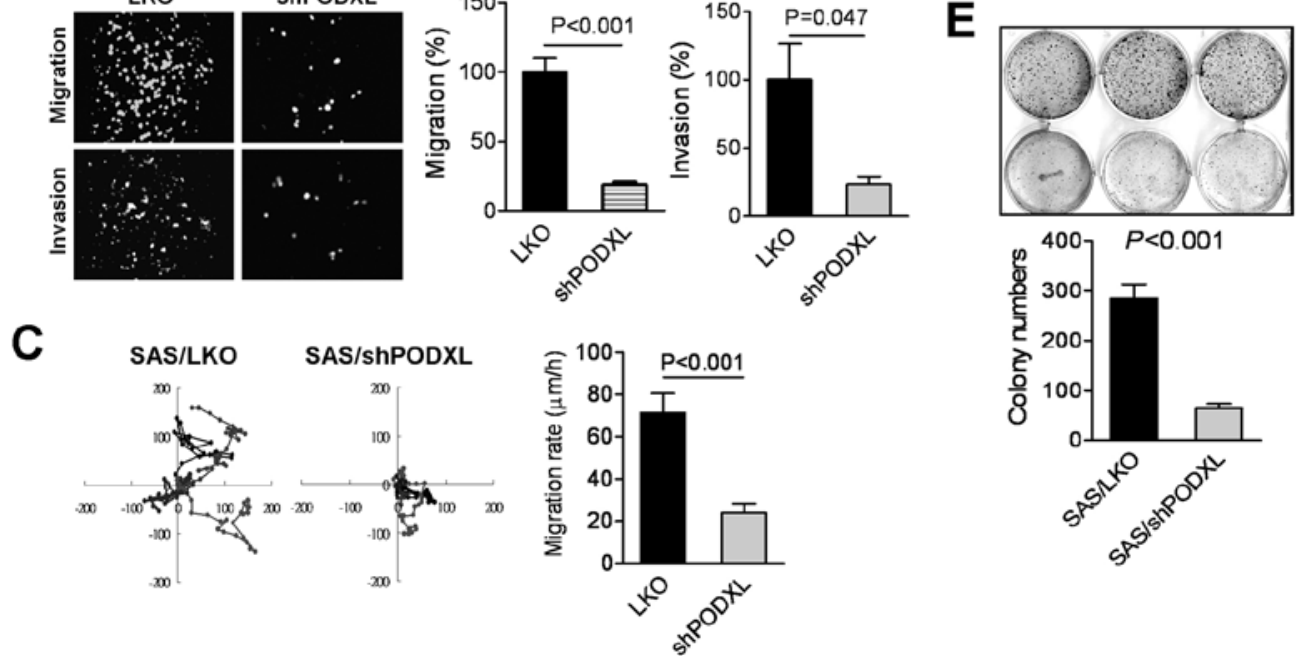

Figure 3. Expression of PODXL regulates cell migration, invasion and proliferation in OSCC. (A) Assessment of PODXL mRNA level and cell migration in OSCC (SAS and FaDu) cells. The expression of PODXL was measured by a real-time PCR, and cell migration was assessed by a Transwell assay. (B) Knockdown of PODXL inhibited cell migration (upper panel) and invasion (lower panel) by SAS cells, as determined by a Transwell assay. (C) Knockdown of PODXL decreased cell motility. Cell migration was tracked and recorded by time-lapse observations. Representative images show the migratory tracks of five cells over $8 \mathrm{~h}$. The migratory velocity was analyzed by calculating the average distance travelled per hour by 20 cells. (D and E) Knockdown of PODXL inhibited tumor growth in SAS cells. Cell proliferation was assessed by (D) an MTT assay, and (E) colony formation assay, as described in Materials and methods. The p-value was determined by an unpaired $t$-test.

We further examined the correlation of PODXL expression with tumor migration in two human oral squamous cancer cell lines, SAS and FaDu. Results of real-time PCR analysis showed a higher expression level of PODXL in SAS cells that exhibited potential migratory ability compared to $\mathrm{FaDu}$ cells (Fig. 3A), suggesting that PODXL expression might be associated with tumor motility. We used shRNA to silence PODXL mRNA in SAS cells, and results of the Transwell analyses showed that the knockdown of PODXL significantly diminished tumor migration and invasion (Fig. 3B). Moreover, time-lapse photographic observations revealed that suppression of PODXL impacted the cell migratory velocity (Fig. 3C). We further found that inhibition of PODXL effectively reduced tumor proliferation and colony formation in SAS cells (Fig. 3D and E), indicating that PODXL expression was associated with tumor aggressiveness.

Suppression of PODXL inhibited FAK activation and filopodia formation. Given that the activation of focal adhesion kinase (FAK) participates in promoting cell migration and invasion, we next assessed whether PODXL regulated FAK signaling. Results showed that the phosphorylation of FAK and its downstream molecule, paxillin, was diminished in PODXL-knockdown SAS cells (Fig. 4A). FAK and paxillin play important roles in modulating F-actin reorganization, which results in enhanced cell polarity. We next examined F-actin expression using phalloidin staining, and the results

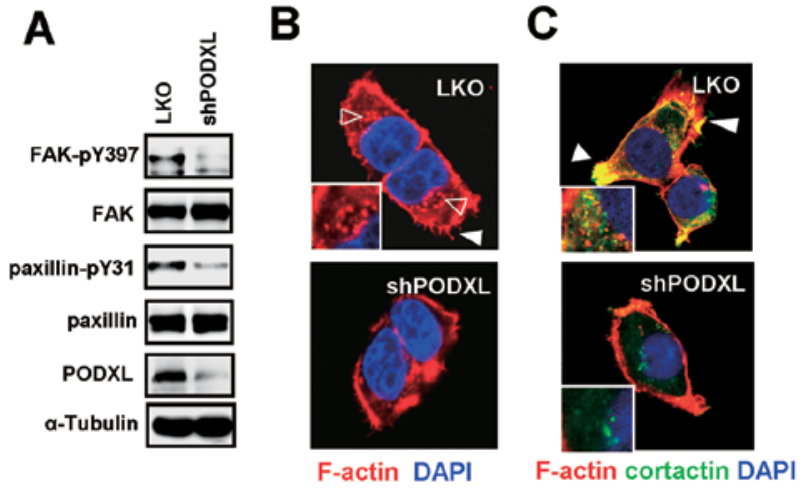

Figure 4. Knockdown of PODXL suppressed focal adhesion kinase (FAK) and paxillin phosphorylation, and colocalization of F-actin and cortactin. (A) Western blot analysis of levels of phosphorylated FAK (pY397) and paxillin (pY31) in PODXL-knockdown SAS cells. (B) Immunofluorescent images of the F-actin structure in PODXL-knockdown cells. F-actin was visualized by rhodamine-conjugated phalloidin staining. Open arrows indicate podosome-like structures, and closed arrows indicate filopodialike structures. The enlarged image shows invadopodia-like punctae. (C) Immunofluorescent analysis of F-actin and cortactin reveals invadopodia. Arrows indicate co-localization of F-actin and cortactin at membrane edges. Enlarged images, co-localization of F-actin and cortactin in the cytoplasm.

showed that suppression of PODXL reduced the expression levels of filopodia at the membrane edge (closed arrow) and podosome-like structures in the perinuclear region 
A

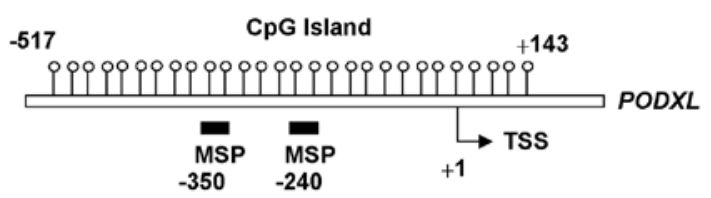

B

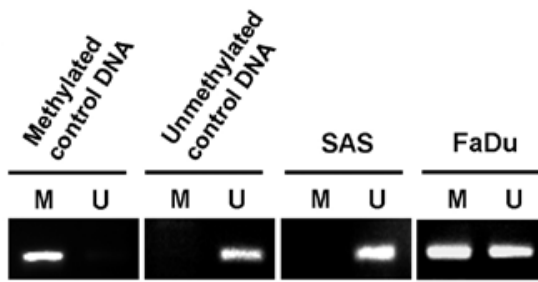

SAS

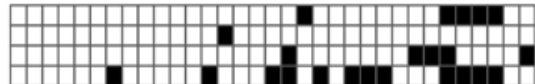

FaDu

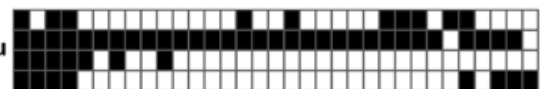

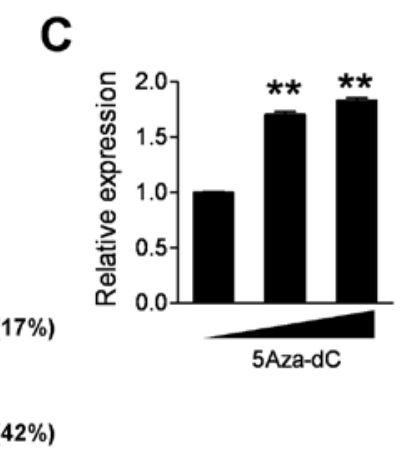

Figure 5. Expression of PODXL is regulated through DNA methylation. (A) Schematic of tentative CpG islands in the PODXL promoter. (B) Analysis of the DNA methylation status in CpG islands of the PODXL promoter by a methyl-specific PCR (MSP), M indicates methylation, U indicates unmethylation (Upper panel). Bisulfate sequencing depicted the tentative methylated site in the PODXL promoter of SAS and FaDu cells (lower panel). An open box indicates unmethylated $\mathrm{CpG}$ dinucleotides, while a closed box indicates methylated ones. Four clones were subjected to DNA sequencing in each cell line, and the percentage of methylated CpG dinucleotides was calculated. (C) Inhibition of DNA methyltransferase increased the PODXL transcriptional level. FaDu cells were treated with 5-aza-2-deoxycytidine (5-aza-dC; $5 \mu \mathrm{M})$ for $72 \mathrm{~h}$, and the level of PODXL mRNA was measured by real-time PCR.

(open arrows), compared to mock-transduced cells (Fig. 4B). To further analyze the effect of PODXL on invadopodia formation, immunofluorescent co-staining with F-actin and cortactin, which were used to identify structures of invadopodia, were performed. Results showed that knockdown of PODXL effectively reduced F-actin and cortactin colocalization. In mock-transduced cells, most of the cortactin and F-actin had co-localized at the membrane edge, indicating the invasive front. Some of the colocalization was observed in the perinuclear region, which indicated the invadopodia precursor (43). However, suppression of PODXL significantly inhibited the colocalization of cortactin and F-actin at both the membrane edge and in perinuclear regions (Fig. 4C), suggesting that inhibition of PODXL impacted focal kinase activation and invadopodia formation.

Expression of PODXL is regulated by DNA methylation. Epigenetic regulation including histone modification and DNA methylation often participates in modulating gene transcription. We next assessed whether the PODXL expression was associated with methylation of its DNA. The MethPrimer database was used to predict a $\mathrm{CpG}$ island located from downstream 143 to upstream 517 of the PODXL gene, which was related to the transcriptional start site (Fig. 5A). Data of the methylate-specific PCR (MSP) assay showed that PODXL displayed totally unmethylated expression in SAS cells, whereas it showed only partially unmethylated status in FaDu cells (Fig. 5B upper panel). Totally unmethylated and methylated genomic DNA was used as experimental controls. Further analysis of the precise methylated level of each $\mathrm{CpG}$ site by a bisulfate sequencing assay showed that PODXL DNA in SAS cells was $17 \%$ methylated, whereas $42 \%$ methylation of the $\mathrm{CpG}$ island of PODXL was detected in FaDu cells (Fig. 5B lower panel). These data were consistent with findings of the real-time PCR and MSP analyses. Moreover, treatment of FaDu cells with 5-aza-deoxycytidine (5-aza-dC) for $72 \mathrm{~h}$, a DNA methyltransferase inhibitor, significant increased the PODXL mRNA level (Fig. 5C), indicating that DNA methylation may participate in regulating PODXL expression.

PODXL modulates ECM and pro-metastatic gene expression levels. To further explore PODXL-mediated gene expression levels, the cDNA of two biological repeats from PODXLknockdown SAS and MDA-MB-231 cells was subjected to microarray analyses to examine gene changes after silencing of PODXL. We found that PODXL suppression markedly attenuated genes specifically associated with ECM organization (COL13, COL17 and ITGB3), cell adhesion and the EMT (CDH1, CDH3, LOX and LOX4), and pro-metastasis cytokines (interleukin (IL) $1 \beta, I L 8$ and $I L 24$ ) (Fig. 6A). The expression levels of these genes were further confirmed by real-time PCR analysis (Fig. 6B). Cellular functional grouping by a Gene Set Enrichment Analysis (GSEA) illustrated that suppression of PODXL significantly affected wound-healing, cell adhesion, ECM polymerization and degradation, and adherent junction assembly (Fig. 6C and D). We further analyzed the hit entities affected by PODXL and linked to the tentative intracellular kinase cascade underlying PODXL using sub-network enrichment analysis (SNEA) algorithm software. The hit entities selected by the microarray data were analyzed by an SNEA algorithm, and the results showed that the pivotal effectors involved in the downstream signaling of PODXL responsible for regulating gene expression, might include Rac1, protein kinase $\mathrm{C}$ (PKC), mitogen-activated protein kinase (MAPK), transforming growth factor (TGF) $\beta$, and activator protein (AP)-1 pathways (Fig. 6E). These data indicated that PODXL plays a crucial role in promoting tumor metastasis. 


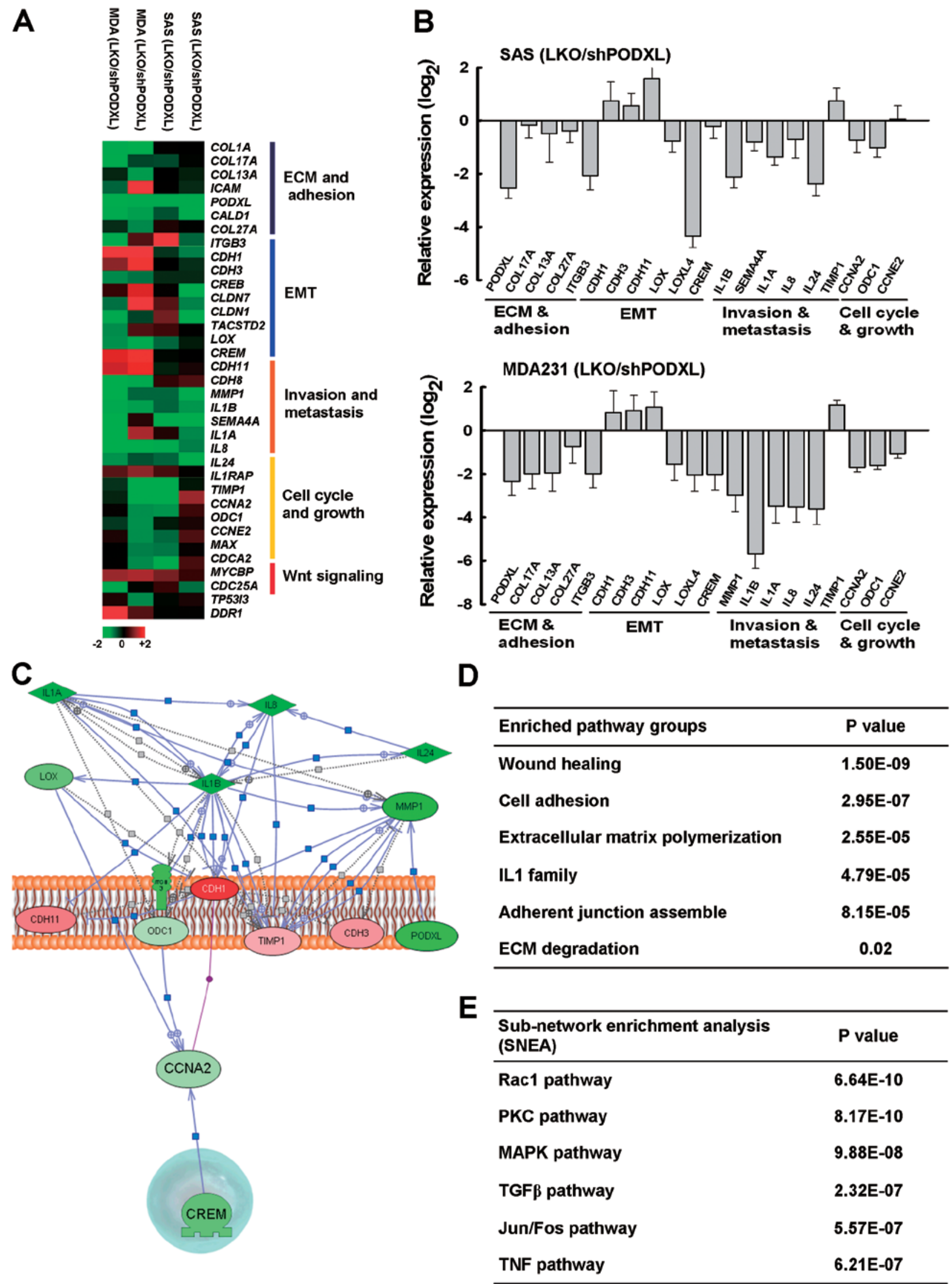

Figure 6. PODXL regulates pro-metastatic gene expression levels. (A) Changes in gene expression levels in SAS and MDA-MB231 cells upon PODXL knockdown, as analyzed by a cDNA microarray. The heat map depicts relative gene expression changes (LKO/shPODXL) from duplicate experiments. (B) Verification of $>2$-fold changes in gene expression identified from the cDNA microarray data by real-time PCR analysis. Representative data show relative expression levels of mock cells by $\log 2$ calculation. (C) A genetic network diagram constructed using Pathway Studio 7 depicting the direct regulation from selected entities (A) affected by PODXL. Green entities indicate downregulation by PODXL, and red entities indicate upregulation. (D) A Gene Set Enrichment Analysis (GSEA) predicted the enriched biological processes affected by PODXL. (E) A Sub-Network Enrichment Analysis (SNEA) algorithm predicted the tentative intracellular signaling cascades mediated by PODXL. 


\section{Discussion}

Overexpression of PODXL was demonstrated to be an independent factor in the poor prognoses of several cancer types such as breast, colon, bladder and brain tumors. However, the role of PODXL in OSCC and its underlying mechanism have not yet been delineated. In the present study, we found that elevation of PODXL correlated with the migratory and invasive abilities of OSCC. Suppression of PODXL significantly diminished oral cancer cell aggressiveness. PODXL silencing inhibited FAK and paxillin activation, and suppressed F-actin and cortactin colocalization. Gene expression profile and molecular pathway analyses revealed that PODXL regulates genes associated with the EMT, ECM polymerization, cell adhesion and metastatic cytokine expression levels. These data suggest that PODXL might play a crucial role in promoting tumor metastases in OSCC.

FAK and its downstream molecule, paxillin, play critical roles in signaling transduction by integrins and the ECM. Activation of FAK and paxillin promotes actin cytoskeletal rearrangement at the leading edge of lamella structures during cell migration. In addition, activation of cortactin participates in actin nucleation, and cortactin colocalization to sites of actin assembly in lamellipodia promotes dynamic cell spreading and motility (44). Colocalization of cortactin with F-actin in the cytoplasm indicates the formation of the invadopodia precursor (45), which further enhances ECM degradation for tumor invasion (46).

Our data showed that knockdown of PODXL inhibited activation of FAK and paxillin, and suppressed colocalization of cortactin with actin both at the membrane edge and in the cytoplasm. Phalloidin staining data also showed that knockdown of PODXL reduced the formation of filopodia and podosome-like punctae, indicating that PODXL is crucial for cell mobility. Moreover, a gene analytical profile revealed that suppression of PODXL significantly affected a cohort of genes associated with ECM organization $(C O L 13, C O L 17$ and ITGB3), cell adhesion and the EMT ( $C D H 1, C D H 3, L O X$ and $L O X 4)$, and pro-metastasis cytokines (ILI $\beta$, IL8 and IL24). The induction of matrix metalloproteinase (MMP)-9 by PODXL was also identified in MCF7 breast cancer cells (9), suggesting that PODXL predominantly regulates tumor invasiveness. In addition to regulating intracellular signaling by PODXL, the extracellular domain of PODXL is enriched in sialofucosylated oligosaccharides, which both serve as O-linked glycans to bind $\mathrm{E}$ - and L-selectin $(47,48)$, and facilitate interactions of circulating tumor cells and the vasculature during tumor metastasis. Together, the evidence highlights the importance of PODXL in tumor invasiveness and metastases.

Aberrant DNA methylation was demonstrated to be associated with cancer formation. Deciphering abnormalities in DNA methylation would be conducive to understanding the early onset of neoplastic progression. A global analysis of DNA methylation in oral cancer revealed that increased DNA hypermethylation was detected in dysplasia, compared to normal tissues (20). Both DNA hypomethylation and hypermethylation were changed more frequently in oral carcinoma in situ (OIC/OSCC), suggesting that epigenetic deregulation is more prevalent in OSCC progression. The most commonly reported changes in OSCC are the hypermethylation of
E-cadherin (CDH1), PTEN, and p16 (CDKN2A) (49-51). Very few studies delineated DNA hypomethylation in OSCC. We found that the PODXL promoter region from -517 to +143 exhibited abundant $\mathrm{CpG}$ dinucleotides. Highly invasive SAS cells showed hypomethylation in the PODXL promoter region, whereas lowly invasive $\mathrm{FaDu}$ cells showed half methylation. Treatment of low PODXL-expressing cells with 5-aza-dC, a DNA methyltransferase inhibitor, increased the PODXL transcription level. A previous study reported that transcriptional regulation of PODXL is supported by the Sp1 transcription factor (52) and that binding of Sp1 to DNA interferes with DNA methylation (53). However, it is still unclear whether the hypomethylation of PODXL DNA is regulated by Sp1 binding or by other passive DNA demethylation processes. Nevertheless, our data suggest that hypomethylation of the PODXL promoter is associated with cell invasiveness and can be used as a diagnostic biomarker for OSCC.

In conclusion, our results showed that elevation of PODXL is associated with tumor aggressiveness through FAK/paxillin/ cortactin signaling induction and metastatic gene expression level promotion in OSCC, suggesting its clinical value as a prognostic biomarker and as a therapeutic target for managing metastatic OSCC.

\section{Acknowledgements}

We thank the Core Facility of the Institute of Cellular and Organismic Biology, Microarray Core Facility of the Institute of Molecular Biology, and the RNAi Core, Academia Sinica for their technical support. This study was supported by grants from Academia Sinica and the National Science Council (NSC101-2321-B-001-021 and NSC102-2325-B-001-010 to H.-C.W.) and (NSC102-2320-B-038-005 to C.-W.L.).

\section{References}

1. Chen YJ, Chang JT, Liao CT, et al: Head and neck cancer in the betel quid chewing area: recent advances in molecular carcinogenesis. Cancer Sci 99: 1507-1514, 2008.

2. Zygogianni AG, Kyrgias G, Karakitsos P, et al: Oral squamous cell cancer: early detection and the role of alcohol and smoking. Head Neck Oncol 3: 2, 2011.

3. Fina L, Molgaard HV, Robertson D, et al: Expression of the CD34 gene in vascular endothelial cells. Blood 75: 2417-2426, 1990.

4. Labastie MC, Cortes F, Romeo PH, Dulac C and Peault B: Molecular identity of hematopoietic precursor cells emerging in the human embryo. Blood 92: 3624-3635, 1998.

5. Kerjaschki D, Sharkey DJ and Farquhar MG: Identification and characterization of podocalyxin - the major sialoprotein of the renal glomerular epithelial cell. J Cell Biol 98: 1591-1596, 1984.

6. Doyonnas R, Nielsen JS, Chelliah S, et al: Podocalyxin is a CD34-related marker of murine hematopoietic stem cells and embryonic erythroid cells. Blood 105: 4170-4178, 2005.

7. Nielsen JS and McNagny KM: The role of podocalyxin in health and disease. J Am Soc Nephrol 20: 1669-1676, 2009.

8. Economou CG, Kitsiou PV, Tzinia AK, et al: Enhanced podocalyxin expression alters the structure of podocyte basal surface. J Cell Sci 117: 3281-3294, 2004.

9. Sizemore S, Cicek M, Sizemore N, Ng KP and Casey G: Podocalyxin increases the aggressive phenotype of breast and prostate cancer cells in vitro through its interaction with ezrin. Cancer Res 67: 6183-6191, 2007.

10. Hsu YH, Lin WL, Hou YT, et al: Podocalyxin EBP50 ezrin molecular complex enhances the metastatic potential of renal cell carcinoma through recruiting Racl guanine nucleotide exchange factor ARHGEF7. Am J Pathol 176: 3050-3061, 2010. 
11. Schmieder S, Nagai M, Orlando RA, Takeda T and Farquhar MG: Podocalyxin activates RhoA and induces actin reorganization through NHERF1 and Ezrin in MDCK cells. J Am Soc Nephrol 15: 2289-2298, 2004.

12. Doyonnas R, Kershaw DB, Duhme C, et al: Anuria, omphalocele, and perinatal lethality in mice lacking the CD34-related protein podocalyxin. J Exp Med 194: 13-27, 2001.

13. Forse CL, Yilmaz YE, Pinnaduwage D, et al: Elevated expression of podocalyxin is associated with lymphatic invasion, basal-like phenotype, and clinical outcome in axillary lymph node-negative breast cancer. Breast Cancer Res Treat 137: 709-719, 2013.

14. Binder ZA, Siu IM, Eberhart CG, et al: Podocalyxin-like protein is expressed in glioblastoma multiforme stem-like cells and is associated with poor outcome. PLoS One 8: e75945, 2013.

15. Boman K, Larsson AH, Segersten U, et al: Membranous expression of podocalyxin-like protein is an independent factor of poor prognosis in urothelial bladder cancer. Br J Cancer 108 2321-2328, 2013

16. Larsson A, Johansson ME, Wangefjord S, et al: Overexpression of podocalyxin-like protein is an independent factor of poor prognosis in colorectal cancer. Br J Cancer 105: 666-672, 2011.

17. Chen QW, Zhu XY, Li YY and Meng ZQ: Epigenetic regulation and cancer (Review). Oncol Rep 31: 523-532, 2014.

18. Jithesh PV, Risk JM, Schache AG, et al: The epigenetic landscape of oral squamous cell carcinoma. Br J Cancer 108: 370-379, 2013

19. Huang TT, Gonzales CB, Gu F, et al: Epigenetic deregulation of the anaplastic lymphoma kinase gene modulates mesenchymal characteristics of oral squamous cell carcinomas. Carcinogenesis 34: 1717-1727, 2013

20. Towle R, Truong D, Hogg K, Robinson WP, Poh CF and Garnis C: Global analysis of DNA methylation changes during progression of oral cancer. Oral Oncol 49: 1033-1042, 2013.

21. Hong Y, Downey T, Eu KW, Koh PK and Cheah PY: A 'metastasis-prone' signature for early-stage mismatch-repair proficient sporadic colorectal cancer patients and its implications for possible therapeutics. Clin Exp Metastasis 27: 83-90, 2010.

22. Kaiser S, Park YK, Franklin JL, et al: Transcriptional recapitulation and subversion of embryonic colon development by mouse colon tumor models and human colon cancer. Genome Biol 8: R131, 2007.

23. Ki DH, Jeung HC, Park $\mathrm{CH}$, et al: Whole genome analysis for liver metastasis gene signatures in colorectal cancer. Int J Cancer 121: 2005-2012, 2007.

24. Sabates-Bellver J, Van der Flier LG, de Palo M, et al Transcriptome profile of human colorectal adenomas. Mol Cancer Res 5: 1263-1275, 2007.

25. Skrzypczak M, Goryca K, Rubel T, et al: Modeling oncogenic signaling in colon tumors by multidirectional analyses of microarray data directed for maximization of analytical reliability. PLoS One 5: pii: e13091, 2010.

26. Cho JY, Lim JY, Cheong JH, et al: Gene expression signaturebased prognostic risk score in gastric cancer. Clin Cancer Res 17: 1850-1857, 2011

27. D'Errico M, de Rinaldis E, Blasi MF, et al: Genome-wide expression profile of sporadic gastric cancers with microsatellite instability. Eur J Cancer 45: 461-469, 2009.

28. Wang Q, Wen YG, Li DP, et al: Upregulated INHBA expression is associated with poor survival in gastric cancer. Med Oncol 29: 77-83, 2012.

29. Chen X, Cheung ST, So S, et al: Gene expression patterns in human liver cancers. Mol Biol Cell 13: 1929-1939, 2002.

30. Mas VR, Maluf DG, Archer KJ, et al: Genes involved in viral carcinogenesis and tumor initiation in hepatitis $\mathrm{C}$ virus-induced hepatocellular carcinoma. Mol Med 15: 85-94, 2009.

31. Roessler S, Jia HL, Budhu A, et al: A unique metastasis gene signature enables prediction of tumor relapse in early-stage hepatocellular carcinoma patients. Cancer Res 70: 10202-10212, 2010

32. Wurmbach E, Chen YB, Khitrov G, et al: Genome-wide molecular profiles of HCV-induced dysplasia and hepatocellular carcinoma. Hepatology 45: 938-947, 2007.

33. $\mathrm{Su} \mathrm{H}, \mathrm{Hu} \mathrm{N}$, Yang $\mathrm{HH}$, et al: Global gene expression profiling and validation in esophageal squamous cell carcinoma and its association with clinical phenotypes. Clin Cancer Res 17: 2955-2966, 2011.
34. Hao Y, Triadafilopoulos G, Sahbaie P, Young HS, Omary MB and Lowe AW: Gene expression profiling reveals stromal genes expressed in common between Barrett's esophagus and adenocarcinoma. Gastroenterology 131: 925-933, 2006.

35. Hu N, Clifford RJ, Yang HH, et al: Genome wide analysis of DNA copy number neutral loss of heterozygosity (CNNLOH) and its relation to gene expression in esophageal squamous cell carcinoma. BMC Genomics 11: 576, 2010.

36. Kim SM, Park YY, Park ES, et al: Prognostic biomarkers for esophageal adenocarcinoma identified by analysis of tumor transcriptome. PLoS One 5: e15074, 2010.

37. Kimchi ET, Posner MC, Park JO, et al: Progression of Barrett's metaplasia to adenocarcinoma is associated with the suppression of the transcriptional programs of epidermal differentiation. Cancer Res 65: 3146-3154, 2005.

38. Cromer A, Carles A, Millon R, et al: Identification of genes associated with tumorigenesis and metastatic potential of hypopharyngeal cancer by microarray analysis. Oncogene 23 : 2484-2498, 2004.

39. Ginos MA, Page GP, Michalowicz BS, et al: Identification of a gene expression signature associated with recurrent disease in squamous cell carcinoma of the head and neck. Cancer Res 64: $55-63,2004$

40. Schlingemann J, Habtemichael N, Ittrich C, et al: Patient-based cross-platform comparison of oligonucleotide microarray expression profiles. Lab Invest 85: 1024-1039, 2005.

41. Sengupta S, den Boon JA, Chen IH, et al: Genome-wide expression profiling reveals EBV-associated inhibition of MHC class I expression in nasopharyngeal carcinoma. Cancer Res 66: 7999-8006, 2006.

42. Toruner GA, Ulger C, Alkan M, et al: Association between gene expression profile and tumor invasion in oral squamous cell carcinoma. Cancer Genet Cytogenet 154: 27-35, 2004.

43. Murphy DA and Courtneidge SA: The 'ins' and 'outs' of podosomes and invadopodia: characteristics, formation and function. Nat Rev Mol Cell Biol 12: 413-426, 2011.

44. Sung BH, Zhu X, Kaverina I and Weaver AM: Cortactin controls cell motility and lamellipodial dynamics by regulating ECM secretion. Curr Biol 21: 1460-1469, 2011.

45. Oser M, Yamaguchi H, Mader CC, et al: Cortactin regulates cofilin and N-WASp activities to control the stages of invadopodium assembly and maturation. J Cell Biol 186: 571-587, 2009.

46. Clark ES, Whigham AS, Yarbrough WG and Weaver AM: Cortactin is an essential regulator of matrix metalloproteinase secretion and extracellular matrix degradation in invadopodia. Cancer Res 67: 4227-4235, 2007.

47. Dallas MR, Chen SH, Streppel MM, Sharma S, Maitra A and Konstantopoulos K: Sialofucosylated podocalyxin is a functional E- and L-selectin ligand expressed by metastatic pancreatic cancer cells. Am J Physiol Cell Physiol 303: C616-C624, 2012.

48. Thomas SN, Schnaar RL and Konstantopoulos K: Podocalyxin-like protein is an E-/L-selectin ligand on colon carcinoma cells: comparative biochemical properties of selectin ligands in host and tumor cells. Am J Physiol Cell Physiol 296: C505-C513, 2009.

49. Su PF, Huang WL, Wu HT, Wu CH, Liu TY and Kao SY: p16(INK4A) promoter hypermethylation is associated with invasiveness and prognosis of oral squamous cell carcinoma in an age-dependent manner. Oral Oncol 46: 734-739, 2010.

50. Alyasiri NS, Ali A, Kazim Z, et al: Aberrant promoter methylation of PTEN gene among Indian patients with oral squamous cell carcinoma. Int J Biol Markers 28: 298-302, 2013.

51. Viswanathan M, Tsuchida N and Shanmugam G: Promoter hypermethylation profile of tumor-associated genes p16, p15, hMLH1, MGMT and E-cadherin in oral squamous cell carcinoma. Int J Cancer 105: 41-46, 2003.

52. Butta N, Larrucea S, Alonso S, et al: Role of transcription factor Sp1 and $\mathrm{CpG}$ methylation on the regulation of the human podocalyxin gene promoter. BMC Mol Biol 7: 17, 2006.

53. Cao YX, Jean JC and Williams MC: Cytosine methylation of an Sp1 site contributes to organ-specific and cell-specific regulation of expression of the lung epithelial gene tlalpha. Biochem J 350: 883-890, 2000. 